Vikas Dhikav ${ }^{1 *}$, Dolly Prajapati ${ }^{2}$, Wasifa Muzaffar ${ }^{2}$, Nidhi Agarwal' ${ }^{2}$, Sekh Raisuddin $^{2}$, Kuljeet Singh Anand ${ }^{1}$

${ }^{1}$ Department of Neurology, Postgraduate Institute of Medical Education \& Research (PGIMER), Dr. Ram Manohar Lohia Hospital, New Delhi, India ${ }^{2}$ Center for Translational Research, Jamia Hamdard University, New Delhi, India

Dates: Received: 02 November, 2016; Accepted: 02 January, 2017; Published: 03 January, 2017

*Corresponding author: Dr. Vikas Dhikav, Department of Neurology, PGIMER \& Dr. RML Hospital, New Delhi, \#505, 5th floor, PGIMER (Admin) Block, 110001, India, Tel: +91 9868953977; 011-23741635; E-mail: vikasdhikav@hotmail.com www.peertechz.com

Keywords: Amisulpride; Effectiveness; Antipsychotics; Safety; Tolerability; BPSD

\author{
Research Article
}

\section{Amisulpride in Refractory Behavioral and Psychological Symptoms of Dementia}

\begin{abstract}
Background: Amisulpiride is an atypical antipsychotic used extensively in Schizophrenia and related disorders. The current study explored the safety and tolerability of amisulpride in refractory patients with behavioral and psychological symptoms of dementia (BPSD), especially in agitation and/ or aggression.

Method: A 12-weeks observational study was conducted in Northern India. A Total of 39 subjects with dementias receiving antipsychotics divided into 3 groups: 19 patients on amisulpride, 10 patients on other anti-psychotics (olanzapine, quietiapine and risperidone), 10 patients not on any antipsychotics. A total of 10 healthy volunteers were taken as controls $(n=49)$. The effectiveness of the antipsychotics in BPSD was evaluated by caregiver rated Likert scale. Agitated Behavior Scale (ABS) was used for categorization of nature of BPSD. Safety and tolerability was assessed using Naranjo Adverse Drug Reaction Probability Scale.

Results: A total of 39 subjects [M: $F=29: 10]$ with mean age $70 \pm 8.1$ years, and with mean duration of illness $4.2 \pm 2.5$ years were recruited. The Mini Mental State Examination (MMSE) was $9.13 \pm 8.5$. A comparison of ABS scores using Analysis of Variance (ANOVA) showed all groups were statistically different $(p<0.0001)$. The Agitated Behavior Scale Scores (ABS scores) were significantly different when groups on antipsychotics were compared with the BPSD patients not on antipsychotic $(p<0.001)$. ABS scores comparisions using paired t-test showed that the amisulpride group was significantly different before and after the amisulpride treatment $(p<0.0001)$. ABS scores and MMSE had a statistically significant correlation (Pearson correlation coefficient $=0.41$ ).

Conclusions: Amisulpride $100 \mathrm{mg} /$ day was safe and well tolerated in patients with refractory BPSD especially in patients with aggression and/or agitation. The tolerability was equivalent to other antipsychotics used routinely in BPSD variety. The self-rated caregiver rating of antipsychotic effectiveness using Likert scale showed the amisulpride had a comparable effectiveness with that of other antipsychotics used routinely in dementias with BPSD.
\end{abstract}

\section{Introduction}

Dementia is a common disease of the elderly and is characterized by cognitive and non-cognitive complaints [1]. Dementias involves loss of cognitive abilities and at least one of the following e.g., aphasia, apraxia, agnosia, disturbances in executive functioning [2]. The behavioural impairment in dementia interferes with a person's Activity of Daily Living [3]. It is a common cause of caregiver stress and burden and leads to poor Quality of Life [4]. Psychosis is the most common disturbance in patients with dementias with behavioral and psychological symptoms of dementia (BPSD) [5], which affects up to $90 \%$ of all dementia subjects [6]. Psychotic BPSD include verbal and physical aggression, agitation, hallucinations delusions, irritability, sleep disturbances, oppositional behaviors, and wandering type behaviors [7] etc. Course of dementias is complicated by the occurrence of BPSD [1] and is the single most predictor of hospitalization and caregiver stress [5].

Currently, the antipsychotics are the most commonly offlabel drugs used in the management of BPSD [8]. These have been the mainstay of the treatment of BPSD, especially the psychotic behaviours. The commonly used drugs for this purpose include olanzapine, quetiapine and risperidone. There are many patients who fail to respond to these drugs [9], leaving a window for the new entry [1]. Since a sizable number of patients do not adequately respond to the available drugs [1,9-12], drugs such as ramelteon [9] and carbamazepine [11] and blonanserin [12], have been tried. Even some radical treatments such as electroconvulsive treatment (ECT) and positive pressure ventilation [13], have been suggested [1], due to constraints in the use of available antipsychotics in BPSD [13].

Though both typical and atypical antipsychotics have been used for management of BPSD; atypical antipsychotics [14,15], are commonly used in BPSD or in Mild Cognitive Impairments (MCI) [15], due to their lower incidence of adverse drug reactions (ADRs) compared to typical ones. The main difference between the two is in terms of their side effect profile specially with respect to extrapyramidal symptoms (EPS) such as acute dystonia, akathisia, parkinsonism and tardive dyskinesia [14], etc. It has been shown that antipsychotic usage can and reduce caregiver burden [16].

Amisulpride is a second generation atypical antipsychotic that selectively binds to dopamine $\mathrm{D}_{2} / \mathrm{D}_{3}$ receptors with $5-\mathrm{HT}_{7}$ antagonistic effect in the limbic system of the brain [14,15]. Amisulpride has not 
been compared in terms of its safety and tolerability with other antipsychotics due to lack of head to head comparative studies [16]. Also there are very limited options available to treat refractory patients with BPSD. The present study was therefore planned to know safety and tolerability of amisulpride in refractory BPSD especially in agitation and/or aggression. An attempt was made to compare the same with commonly used antipsychotics in BPSD. A caregiver rated Likert Rating (out of 10) was used to know the effectiveness of the drug in agitation and/or aggression upon open-label prescription of the drug for refractory patients with BPSD.

\section{Method}

\section{Screening}

A total 300 patients with memory and/or cognitive complaints came to the outpatient department of Super-Specialty Memory Clinic at a tertiary care in Northern India during the study period.

Patients with subjective memory complaints underwent detailed clinical, neurological and neuropsychological evaluation. Those patients with a diagnosis of dementia and refractory BPSD were enrolled in the study for detailed evaluation. Refractory BPSD [12], was defined as patients who had been prescribed more than two kinds of antipsychotic drugs and in whom the drugs were discontinued, because they were either ineffective or the side effects appeared. Each drug was prescribed independently for at least 2 weeks.

Patients who met the diagnostic criteria for dementia (DSMIV) [16] and with BPSD were on amisulpiride $(n=19)$, and other antipsychotics $(\mathrm{n}=10)$. For comparison, the demented controls not on antipsychotics $(n=10)$ were enrolled in the present study. A total of 10 healthy controls were included for comparison (total $n=49$ ).

A total of 64 subjects underwent clinical evaluation and only 39 subjects met the diagnostic criteria (DSM-IV) for diagnosis of dementia. Once the diagnostic evaluation was done, the selected patients were evaluated for recording ADR data. A total of 10 healthy controls were recruited. So a total of 49 subjects $(\mathrm{M}: \mathrm{F}=39: 10)$ were divided into four groups, 19 patients on amisulpride (Group-I), 10 patients on other anti-psychotics(Group-II), 10 patients not on any anti-psychotics (Group-III)and 10 healthy volunteers groups(GroupIV). The excluded patients had BPSD of affective nature (e.g. depression, excessive crying, apathy etc) or refused to enter into the study $(n=25)$.

All patients taking amisulpride were on $100 \mathrm{mg} / \mathrm{daily}$ dose, while patients on other antipsychotics $(n=2)$ were taking olanzapine (5mg/daily). Dose of quitiapine taken by patients $(\mathrm{n}=6)$ was $25 \mathrm{mg}$ and patients on rispridone ( $\mathrm{n}=2$ ) were taking $0.5 \mathrm{mg}$ and $1 \mathrm{mg}$ daily doses respectively. Patients who were prescribed amisulpiride were the ones who were refractory to available drugs but the ones on other antipsychotics were treatment naive patients.

\section{Inclusion and exclusion criteria}

Patients with a diagnosis of dementia using DSM-IV taking antipsychotics and who were refractory to either 2 or more of the three commonly used antipsychotics (quietiapine, risperidone and olanzapine) given for at least 2-weeks were included in the present study.

Attendants of the refractory patients were interviewed thoroughly to know about the drug efficacy in terms of their effects on behavior, on verbal or physical agitation/aggression etc. if the attendants rated the drug response poor on a Likert Scale of $10(<3)$; were recruited in the present study.

Patients who were having BPSD but were treatment naïve were selected as controls. A total of 10 age matched cognitively normal subjects $>60$ years were taken as healthy controls (mean age $=62.4 \pm 1.4$ years). Patients with a diagnosis of dementia but having non-psychotic variety of BPSD e.g. affective disturbances etc. were excluded from the present study. Written and informed consent was taken from all study participants. The study was approved by institutional Ethics Committee.

\section{Control group}

A total 10 healthy volunteers above age 60 years (M:F=7:3; $62.4 \pm 1.4$ years) were recruited in the study. Demographic details like age, sex, and educational background was noted.

\section{Safety and tolerability assessment}

Naranjo's scale was used for the monitoring of Adverse Drug Reactions. Patients were assessed at baseline (day-0), and at the end of study period (end of 12 weeks).

\section{Study design}

It was a 12 -weeks, observational study was conducted in Indian demented patients with refractory BPSD in a Tertiary Care setting. Patients with dementia with BPSD who were refractory to at least 2 of the antipsychotics were observed for safety and tolerability when they were given amisulpride. An attempt was made to assess the effectiveness amisulpride group using self-rated caregiver rating (Likert scale).

\section{Diagnostic and rating scales}

Diagnostic \& Statistical Manual-IV criteria (DSM-IV criteria) was used to diagnosis multiple cognitive deficits which are manifested by both memory impairment and cognitive disturbances. According to this criteria, dementia is a syndrome that may be caused by multiple cognitive deficits, which include memory impairment and at least one of the following: aphasia, apraxia, agnosia, or disturbance in executive functioning.

Mini Mental State Examination (MMSE) [17], was used to test cognitive function among the elderly; it is an 11 questions measure that tests five areas of orientation, attention, memory, severity of language and visual-spatial skills changes over a time. Maximum score is 30 and it categorize global cognitive impairment as par the score mild (27-21), moderate (20-11) and severe $(\leq 10)$. The cut off for dementia is $\leq 24$.

Agitated Behavior Scale (ABS) [18], was used for categorization of BPSD as per ABS scores and patients were classified as mild (2228 ), moderate (29-35) and severe (>36). Agitated behavior scale (ABS) score $>22$ meant absence of clinically significant agitation. 
ABS was developed to assess the nature and extent of agitation during the acute phase of recovery from the brain injury but is applicable to dementias as well. Its primary purpose is to allow serial assessment of agitation by treatment professionals who want objective feedback about the course of a patient's agitation.

Naranjo Adverse Drug Reaction Probability Scale [19], was used for the assessment of safety and tolerability of antipsychotics. An attempt was made to assess the causality using Naranjo Adverse Drug Reaction Probability Scale. The scale consists of 10 questions that are answered either yes, no or don't know. Different point values range from -1 to $+2(-1,0,+1,+2)$. The ADR is divided into definite if the score is 9 or more, probable, if the score is between 5-8, and possible for a score 1-4. Naranjo score is rated 0 for doubtful ADRs.

Likert scale (scores between 0-10) was used to evaluate effectiveness of drugs in BPSD as rated by caregivers, especially for the agitation and/or aggression. Patients were asked to rate the extent of their illness out of a score of 10 and the same question was asked after the duration of drug treatment was over. A before and after comparison was done.

\section{Statistical analysis}

Statistical analysis was done by latest version of Statistical Package for the Social Sciences (SPSS-SPSS Inc., Chicago, IL). Normality of data was checked by using bar graphs. Association between the groups was determined by Pearson Correlation Coefficient. Depending upon the type of variables paired t- test and One-Way Analysis of Variances (ANOVA) was used for comparing means of four groups. The minimum level of significance $(\mathrm{p}<0.05)$ was used to accept or reject null hypothesis. The study was done in accordance with the Indian Council of Medical Research (ICMR, 2006) medical research guidelines involving human subjects. The study was approved by Institutional Ethics Committee.

\section{Results}

Out of the total 300 patients that came in the Neurology Outpatient Department of a Tertiary Care center with memory/ cognitive impairment during the study duration; a total of 64 patients were evaluated in the study duration. Out of this, 49 subjects ( $\mathrm{M}: \mathrm{F}=39: 10)$ were enrolled in the study (Figure 1, Table 1). Most of the enrolled patients were patients with Alzheimer's disease (AD). Others included fronto-temporal dementia, mixed type dementia and vascular dementia. All patients were living in their homes (no hospital resident); the caregivers were usually a family member, especially a son $(n=6)$ or daughter $(n=6)$ or the spouse $(n=26)$, and servant $(n=1)$.

One-Way-ANOVA of 4 independent samples of ABS scores using standard weighted mean analysis was performed and it showed all groups were statistically different $(P<0.0001)$. Tukeys Honestly significant difference (HSD) showed statistically significant differences between the means of all four groups. Paired t-test of ABS scores before and after the amisulpride was significantly different $(p<0.0002)$. ABS scores in both the groups on antipsychotics declined significantly after the study period. The scores were high in group not on any antipsychotics (Figure 2).
Scatter plot between the MMSE and Likert score after amisulpide showed a negative correlation and the Pearson correlation Coefficient (r) was -0.71 . This showed that with decreasing MMSE, the caregiver rated Likert Scale Scores declined implying increasing severity (Figure 3). Likewise, the scatter plot between the MMSE and ABS score after amisulpide treatment showed a modest negative correlation (Pearson correlation coefficient $=-0.41)$. This implied that with increasing severity of dementia (decreasing MMSE), the ABS scores increased (Figure 4).

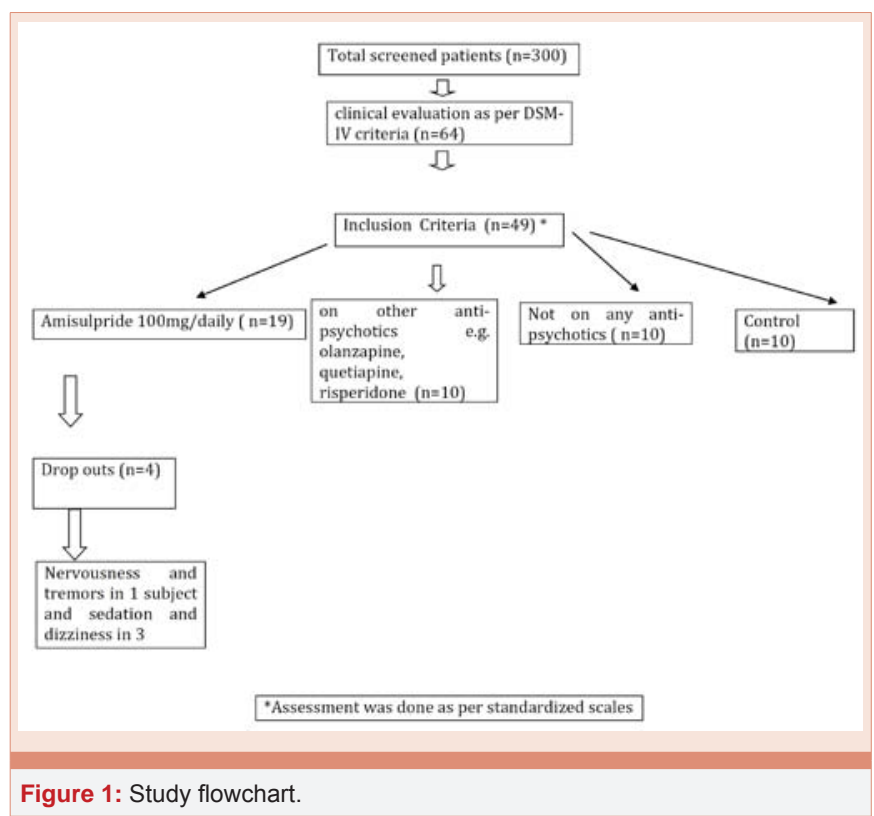

Table1: Demographic details, MMSE and ABS Scores of subjects in the current study.

\begin{tabular}{|l|l|l|l|l|}
\hline Group & $\begin{array}{l}\text { Age } \\
\text { (Mean } \pm \text { SD) } \\
\text { years }\end{array}$ & $\begin{array}{l}\text { Duration } \\
\text { of illness } \\
\text { (mean } \pm \text { SD) } \\
\text { in years }\end{array}$ & $\begin{array}{l}\text { Mean MMSE } \\
\text { scores(mean } \pm \text { SD) }\end{array}$ & $\begin{array}{l}\text { Mean ABS } \\
\text { score } \\
\text { before } \\
\text { drugs } \\
\text { (mean } \pm S D)\end{array}$ \\
\hline Amisulpride & $70.23 \pm 20.12$ & $3.50 \pm 2.21$ & $9.1 \pm 8.22$ & $42.26 \pm 12$ \\
\hline $\begin{array}{l}\text { Other } \\
\text { antipsychotics }\end{array}$ & $65.12 \pm 15.11$ & $4.12 \pm 2.15$ & $10.2 \pm 8.36$ & $39.26 \pm 12$ \\
\hline $\begin{array}{l}\text { No } \\
\text { antipsychotics }\end{array}$ & $62.16 \pm 18.71$ & $2.22 \pm 2.11$ & $15.1 \pm 8.52$ & $35.0 \pm 8.17$ \\
\hline Control & $66 \pm 3.5$ & ------ & $28.2 \pm 2.19$ & $16.6 \pm 2.00$ \\
\hline
\end{tabular}

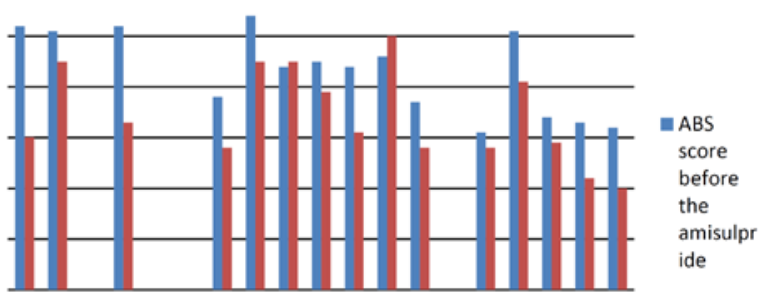

Figure 2: Bar Graph of ABS score before and after the amisulpride treatment. 
After the treatment with amisulpride Likert scores significantly decreased $(\mathrm{P}<0.0001)$ at the end of 12 weeks.

Upon linear regression, the value of coefficient of determination (r2) using scatter plot between the MMSE and ABS score after amisulpide treatment was 0.17 indicating that the chance that the low MMSE explained the observed variances in ABS scores was very small. The observed variance is perhaps due to change in ABS scores.

Safety of antipsychotics was assessed using Naranjo's Algorithm. Patients on amisulpride were able to tolerate the drug for the study duration i.e. 12-weeks $(n=15)$. Discontinuation of the drug was needed in four patients (4/19) due to adverse effects. Nervousness and tremors occurred in one subject and three subjects showed sedation and dizziness. In amisulpiride group, a total 13 patients out of $19(70 \%)$ showed a statistically significant improvement in their ABS scores (Group-I). Two patients remained static with no change. Overall the drug was well tolerated. There were no extra pyramidal Symptoms (EPS) noted during the study. In the antipsychotics (Group-II) 8 out of 10 patients showed a statistically significant change in their ABS scores before and after the treatment $(P<0.0001)$. Overall, the effectiveness of amisulpride was similar to that of other antipsychotics [ $(68 \%$ versus $80 \%$-Odds ratio $=1.1692 ; 95 \%$ confidence interval $=0.3640$ to 3.7562 , $\mathrm{p}$-value $>0.005)$ ].

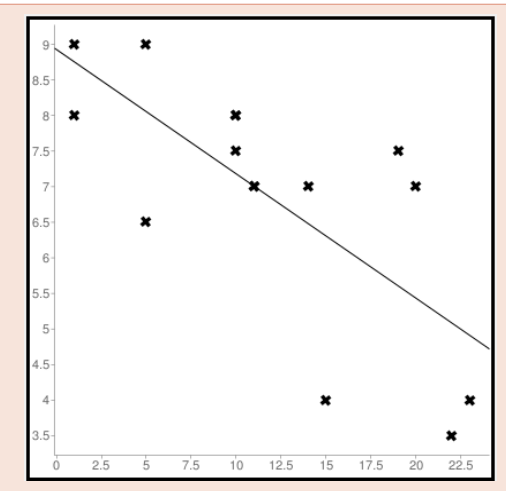

Figure 3: Scatter plot between the MMSE and Likert scores after amisulpide treatment. (Correlation Coefficient ( $r$ ): -0.71). After the treatment with amisulpride Likert scores significantly decreased $(P<0.0001)$ at the end of 12 weeks.

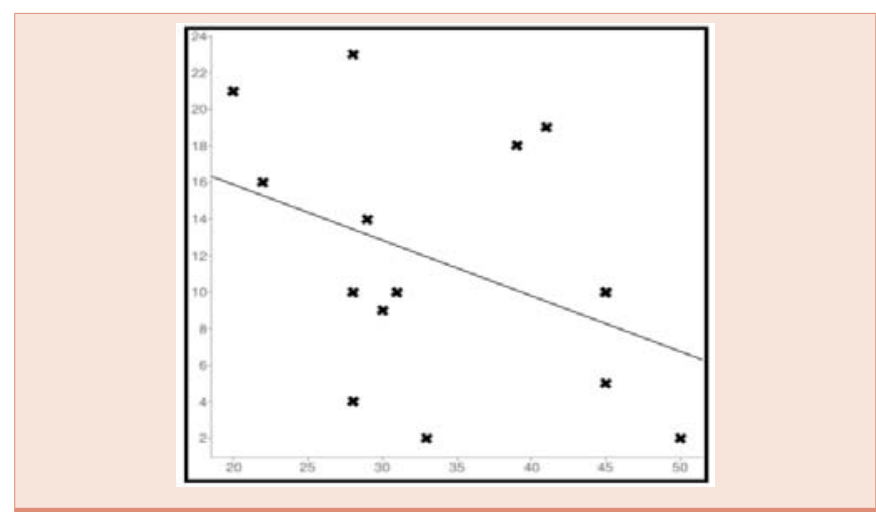

Figure 4: Scatter plot between the MMSE and ABS scores after amisulpide treatment. (Correlation Coefficient (r): -0.41).

\section{Discussion}

BPSD are extremely common in patients with dementias and affect up to $90 \%$ of all dementia subjects [5,6]. Psychotic BPSD include verbal and physical aggression, agitation, hallucinations and delusions, irritability, sleep disturbances, oppositional, and wandering type behaviors7. Compared to the affective BPSD, the psychotic type of BPSD are more troublesome and contribute to caregiver stress, burden and hospitalization [3-5].

Antipsychotics are the most commonly off-label drugs used in the management of BPSD [8] and have been the mainstay of the treatment of BPSD. The common options include olanzapine, quitiapine and risperidone. Anticholinesterases, the primary form of therapies for Alzheimer's disease also have a favourable effect on BPSD [20].

Amisulpride [21-24] is an atypical antipsychotic that has bimodal effects in the brain. The principal effects of amisulpride are related to its ability to modulate $\mathrm{D} 2$ receptors, acting as a modulator of D2 receptors at low dose and blocker at high dose. The drug acts as an antidepressant in low dose and as antipsychotic at high dose. The drug has pharmacological effects in nigrostriatal and mesolimbic pathways in the brain. The pharmacological effects of this drug have been confirmed using positron emission tomography.

Amisulpride is commonly used in acute and chronic schizophrenia. The drug in adult patients is given in the dose of 400$1200 \mathrm{mg}$ daily but a lower dose between $50-200 \mathrm{mg}$ is given in elderly. The lower dose works well for negative symptoms in adults as well.

Though a variety of pharmacological and non-pharmacological treatment options have been recommended for the treatment BPSD; antipsychotics are most commonly used [25]. One important reason of their widespread use is their better efficacy compared to nonpharmacological methods [26]. No conclusive evidence exists to show that antipsychotics have an alternative in terms of their safety for BPSD [26].

Despite the common use of antipsychotics for management of BPSD, inappropriate doses and duration are not uncommon [25]. It has been suggested that whatever drug be chosen, it needs to be monitored for first few days and be used in lowest possible dose for shortest duration.

Safety of the antipsychotics has been an issue for patients with dementias and guidelines exist for the use of these drugs. Major side effect of anitpsychotics is extrapyramidal side effects. At dosages less than $300 \mathrm{mg} /$ daily, the incidence of extrapyramidal side effects is equal to that of the placebo with amisulpride [27].

Only one other study to the best of our knowledge has explored the safety and tolerability of amisulpride in BPSD [28]. This small off label study $(n=28)$ had taken patients with dementia of Alzheimer's type and compared amisulpride with risperidone. Present study has screened $>300$ patients and enrolled 49 patients and has taken common types of dementia and not just patients with Alzheimer's disease. Moreover, our group encompasses patients on other commonly used antipsychotics and also healthy controls as well; so present study is more comprehensive evaluation of amisulpride in elderly population. 
Amisulpride has not been investigated in refractory behavioral and psychological symptoms of dementia. Till date, no randomized double blind placebo controlled trials exist for amisulpride in BPSD $[29,30]$.

Though antipsychotics are effective for large number of patients with BPSD; still a large number does not respond to the available drugs [26], (refractory BPSD) and hence there is a need of an antipsychotic drug for these patients. Amisulpride was chosen for this study due to its efficacy, safety and the ease of administration.

A total of $13(70 \%)$ patients improved significantly with amisulpride, however 2 remained static with no change. The discontinuation of drug was needed in 4 patients due to adverse effects. Nervousness and tremors in one subject and 3 subjects showed sedation and dizziness. No serious ADR occurred during the study period. So overall, the drug was well tolerated. ABS and Likert scores declined significantly during 12 -weeks course of treatment with amisulpride. The drug significantly improved agitation and/or aggression.

Assessment and management of refractory BPSD is an essential and challenging part of dementia treatment. This observational study concluded that amisulpride $100 \mathrm{mg}$ /day was safe and well tolerated drug in refractory BPSD. The drug reduced aggression and/or agitation in $70 \%(n=13)$ in demented patients with refractory BPSD without significant side effects. The safety and tolerability of amisulpride was as good as other antipsychotics used routinely in patients with BPSD in usual pharmacological dosages.

\section{Limitations}

The present study has some limitations. First of all, the patients sampled may suffer from severe behavioral disturbances than the average population. Recall bias could affect the generalization like in other studies of this nature. Finally, it was a pilot study so the result would not be generalized for the whole population. More studies at different doses in a large population to confirm the safety, efficacy and tolerability amisulpride in a double blind randomized placebo controlled trials could be done.

\section{Conclusions}

The Results of the present study showed that amisulpride 100 $\mathrm{mg} /$ day is a safe and well tolerated drug for treating demented patients with refractory BPSD, especially for agitation and/or aggression. Amisulpiride could potentially be considered as a pharmacological intervention in the management of refractory BPSD.

\section{References}

1. Kar N (2009) Behavioral and psychological symptoms of dementia and their management. Indian J Psychiatry 51: S77-S86.

2. Ganguli M, Blacker D, Blazer DG, Grant I, Jeste DV, et al. (2011) Classification of Neurocognitive Disorders in DSM-5: A Work in Progress. The American journal of geriatric psychiatry: official journal of the American Association for Geriatric Psychiatry 19: 205-210.

3. Liperoti R, Pedone C, Corsonello A (2008) Antipsychotics for the Treatment of Behavioral and Psychological Symptoms of Dementia (BPSD). Current Neuropharmacology 6: 117-124.
4. Mougias AA, Politis A, Mougias MA, Kotrotsou I, Skapinakis P, et al. (2015) The burden of caring for patients with dementia and its predictors. Psychiatriki 26: 28-37.

5. Dhikav V, Anand KS (2012) Caregiver burden of behavioral and psychological symptoms of dementia among Indian patients with Alzheimer's disease. International Psychogeriatrics 24: 1531-1532.

6. Neil W, Bowie P (2008) Carer burden in dementia assessing the impact of behavioural and psychological symptoms via selfreport questionnaire. Int J Geriatr Psychiatry 23: 60-64.

7. Cerejeira J, Lagarto L, Mukaetova-Ladinska EB (2012) Behavioral and psychological symptoms of dementia. Front Neurol 3: 73.

8. Mauri M, Mancioli A, Rebecchi V, Corbetta S, Colombo C, et al. (2006) Amisulpride in the treatment of behavioural disturbances among patients with moderate to severe Alzheimer's disease. Acta Neurol Scand 114: 97-101.

9. Asano M, Ishitobi M, Tanaka Y, Wada Y (2013) Effects of ramelteon on refractory behavioral and psychological symptoms of dementia in Alzheimer disease. J Clin Psychopharmacol 33: 579-881.

10. Desai AK, Grossberg GT (2001) Recognition and Management of Behavioral Disturbances in Dementia. Prim Care Companion J Clin Psychiatry 3: 93-109.

11. Gleason RP, Schneider LS (1990) Carbamazepine treatment of agitation in Alzheimer's outpatients refractory to neuroleptics. J Clin Psychiatry 51: 115118.

12. Hamuro A, Saito S (2010) Five Alzheimer's disease cases with refractory behavioural psychological symptoms of dementia treated with blonanserin. Psychogeriatrics 10: 198-200.

13. Yamamoto N, Fukuda Y, Shiba H, Ozaki T, Hayashi S, et al. (2012) Noninvasive positive pressure ventilation improved refractory behavioral and psychologicalsymptoms of dementia in an elderly adult with type 2 respiratory failure. J Am Geriatr Soc 60: 1576-1578.

14. David R, Piano J, Robert P (2011) [Treatment of behavioral disorders in Alzheimer's disease]. Rev Prat 61: 939-944.

15. Dhikav V, Sethi M, Mishra P, Singh Anand K (2015) Behavioral and psychological symptoms among Indian patients with mild cognitive impairment. Int Psychogeriatr 27: 2097-2098.

16. Arriola E, Ignacio Diago J, Antonio Burón J, Gallego R (2005) Spanish Risperidone in Dementia Study Group. Open-label, observational study of the effects of risperidone on the behavioral and psychological symptoms ofdementia and caregiver stress in the community setting. Am J Geriatr Pharmacother 3: 8-16.

17. Folstein MF, Folstein SE, McHugh PR (1975) "Mini-mental state". A practical method for grading the cognitive state of patients for the clinician. J Psychiatr Res 12: 189-198.

18. Corrigan JD (1998) Development of a scale for assessment of agitation following traumatic brain injury. J Clin Exp Neuropsychol 11: 261-277.

19. Naranjo CA, Busto U, Sellers EM, Sandor P, Ruiz I, et al. (1981) A method for estimating the probability of adverse drug reactions. Clin Pharmacol Ther 30 : 239-245.

20. Suzuki H, Inoue Y, Mikami K, Gen K (2014) The influence and changes in the dosages of concomitantly used psychotropic drugs associated with the discontinuation of donepezil in severe Alzheimer's disease with behavioral and psychological symptoms on dementia: a preliminary open-label trial. Therapeutic Advances in Psychopharmacology 4: 37-42.

21. Schoemaker H, Claustre Y, Fage D, Rouquier L, Chergui K, et al. (1997) Neurochemical characteristics of amisulpride, an atypical dopamine D2/D3 receptor antagonist with both presynaptic and limbic selectivity. J Pharmacol Exp Ther 280: 83-97.

22. Komossa K, Rummel-Kluge C, Hunger H, Schmid F, Schwarz S, et al. (2010) Amisulpride versus other atypical antipsychotics for schizophrenia. Cochrane Database Syst Rev 1: CD006624. 
23. Lacasse H, Perreault MM, Williamson DR (2006) Systematic review of antipsychotics for the treatment of hospital-associated delirium in medically or surgically ill patients. Ann Pharmacother 40: 1966-1973.

24. Vasilyeva I, Biscontri RG, Enns MW, Metge CJ, Alessi-Severini S (2013) Movement Disorders in Elderly Users of Risperidone and First Generation Antipsychotic Agents: A Canadian Population-Based Study. Amre D, ed. PLoS ONE 8: e64217.

25. Lapeyre-Mestre M (2016) A Review of Adverse Outcomes Associated with Psychoactive Drug Use in Nursing Home Residents with Dementia. Drugs Aging. [Epub ahead of print]

26. Greenblatt HK, Greenblatt DJ (2016) Use of Antipsychotics for the Treatment of Behavioral Symptoms of Dementia. J Clin Pharmacol 56: 1048-1057.
27. Curran MP, Perry CM (2001) Amisulpride: a review of its use in the management of schizophrenia. Drugs 61: 2123-2150.

28. Lim HK, Pae CU, Lee C, Lee CU (2006) Amisulpride versus risperidone treatment for behavioral and psychological symptoms in patients with dementia of the Alzheimer type: a randomized, open, prospective study. Neuropsychobiology 54: 247-251.

29. Ballard C, Waite J (2006) The effectiveness of atypical antipsychotics for the treatment of aggression and psychosis in Alzheimer's disease. Cochrane Database Syst Rev (1): CD003476.

30. Mast G, Fernandes K, Tadrous M, Martins D, Herrmann N, et al. (2016) Persistence of Antipsychotic Treatment in Elderly Dementia Patients: A Retrospective, Population-Based Cohort Study. Drugs Real World Outcomes 3: $175-182$

Copyright: (c) 2017 Dhikav V, et al. This is an open-access article distributed under the terms of the Creative Commons Attribution License, which permits unrestricted use, distribution, and reproduction in any medium, provided the original author and source are credited. 